\title{
Performance Analysis of Feed Forward Broadband Active Noise Control System using Adaptive Algorithms
}

\author{
T. Koteswara rao ${ }^{1}$, B.Bhaskara Rao $^{2}$ \\ PG Student, Dept. of, ECE, Acharya Nagarjuna University College of Engg. \& Tech., Guntur, A.P, India ${ }^{1}$ \\ Assistant Professor, ECE Dept.,ANUCET, Acharya Nagarjuna University, Guntur, A.P, India ${ }^{2}$
}

\begin{abstract}
These algorithms have been applied to an extensive number of problems including noise and echo cancelling, channel equalization, signal prediction, adaptive arrays as well as many others. Noise control is the field of acoustical engineering that deals with reducing unwanted sound in the environment An active noise control (ANC) system based on adaptive filter theory was developed in the 1980s; however, only with the recent introduction of powerful but less expensive digital signal processor (DSP) hardware, such as the TMS320 family, these technologies become practical. These specialized DSPs were designed for real-time numerical processing of digitized signals. These devices have enabled the less-cost implementation of powerful adaptive ANC algorithms and encouraged the widespread development of ANC systems. We are presents general background information about ANC methods. Contrasts between passive and active noise control are mentioned. Different types of noise-control algorithms are discussed: feed forward broadband, feed forward narrowband, and feedback algorithms. In this paper detailed design of a simple ANC system using aTMS320 DSP and the implementation of that design.
\end{abstract}

Keywords: echo cancelling, active noise control, channel equalization, signal prediction

\section{I.INTRODUCTION}

\section{A. The General Concept of Acoustic Noise Control}

Two types of acoustic noise present in the environment. One is caused by turbulence and is totally random. Turbulent noise distributes energy of entire frequency bands. Another type of noise, called narrowband noise, concentrates most of its energy at specific frequencies. There are two types to controlling acoustic noise: passive and active. These active noise control system contains an electro acoustic device that cancels the unwanted sound by generating an anti-sound (anti-noise) of equal amplitude and opposite phase.

\section{B. General Applications of Active Noise Control}

Specific applications for active noise control now under development include attenuation of unavoidable noise sources in the following end-equipment. Noise attenuation is inside passenger compartment and heavy-equipment operator cabin, active engine mount, hands-free cellular phone, etc. Appliance- Single-channel systems: Air conditioning duct, air conditioner, refrigerator, washing machine, furnace, dehumidifier, etc .

\section{TYPES OF ANC SYSTEMS}

Broadband noise cancellation requires knowledge of the noise source(the primary noise) in order to generate the anti-noise signal. The measurement of the primary noise is used as a reference input to the noise canceller. Primary noise correlates with the reference input signal is canceled downstream of the noise generator (a loudspeaker).

When phase and magnitude are correctly modeled in the digital controller. For narrowband noise cancellation, active techniques have been developed that are very effective and that do not rely on causality. Active noise control systems are based on one of two methods. Feed

forward control is where a coherent reference noise input is sensed before it propagates past the canceling speaker.

A. The Broadband Feed forward System

A considerable amount of broadband noise is produced in ducts such as exhaust pipes and ventilation systems. A reference signal $\mathrm{x}(\mathrm{n})$ is sensed by an input microphone close to the noise source before it passes a loudspeaker. The noise canceller uses the reference input signal to generate a signal $\mathrm{y}(\mathrm{n})$ of equal amplitude but $180^{\circ}$ out of phase. The basic principle of the broadband feed forward approach is that the propagation time delay between the upstream noise sensor (input microphone) and the active control source (speaker) offers the opportunity to electrically reintroduce the noise at a position in the field where it will cause cancellation.

\section{ALGORITHMS FOR ANC SYSTEMS}

The most common algorithm applied to adaptive filters is the transversal filter using the least mean-Squared (LMS) algorithm. The effort in obtaining fast versions of more complex algorithms results from the desire of reducing the computational requirements to a minimal number of operations, as well as reducing the size of memory necessary to run these algorithms in practical applications. The output signal is compared to a second signal d(n), called the desired response signal, by subtracting the two samples at time $n$. This difference signal, given by

$$
e(n)=d(n)-y(n)
$$

$\mathrm{e}(\mathrm{n})$ is known as the error signal. The error signal is fed into a procedure which alters or adapts the parameters of the filter from time $n$ to time $(n+1)$ in a well-defined manner. 


\section{A.LMS Algorithm}

The LMS algorithm based on the steepest-descent method using the negative gradient of the instantaneous squared error, i.e. $J \approx e^{2}(n)$, was devised by Widrow and Stearns to study the pattern-recognition machine. The LMS algorithm updates the weight vector as follows:

$$
\boldsymbol{w}(n+1)=\boldsymbol{w}(n)+\mu e(n) x(n)
$$

It has been shown that the stability of the algorithm requires a more stringent condition on the upper bound of $\mu$ when convergence of the weight variance is imposed. For Gaussian signals, convergence of the MSE requires 0 $<\mu<2 / 3 M P x$.

\section{B.NLMS Algorithm}

Most parameters of the NLMS algorithm are the same as the LMS algorithm, except that the step size mu is now bounded between 0 and 2. The NLMS algorithm includes an additional normalization term $\mathbf{x}^{T}(\mathrm{n}) \mathbf{x}(\mathrm{n})$, as shown in the following equation:

$$
\boldsymbol{w}(n+1)=\boldsymbol{w}(n)+\mu \frac{x(n)}{x^{T}(n) x(n)} e(n)
$$

It makes the convergence rate independent of signal power by normalizing the input vector $\mathbf{u}(n)$ with the energy $x^{T}(n) x(n)=\sum_{m=0}^{M-1} x^{2}(n-m)$ of the input signal in the adaptive filter.

\section{Filtered-X Least-Mean-Square (FXLMS) Algorithm}

The ideal active noise control system uses an adaptive filter $\mathrm{w}(\mathrm{z})$ to estimate the response of an unknown primary acoustic path $\mathrm{P}(\mathrm{z})$ between the reference input sensor and the error sensor. The z-transform of e(n) can be expressed as

$$
\mathrm{E}(\mathrm{z})=\mathrm{D}(\mathrm{z})+\mathrm{Y}(\mathrm{z})=\mathrm{X}(\mathrm{z})[\mathrm{P}(\mathrm{z})+\mathrm{W}(\mathrm{z})]
$$

Where $E(z)$ is the error signal, $X(z)$ is the input signal, and $\mathrm{Y}(\mathrm{z})$ is the adaptive filter output. After the adaptive filter $\mathrm{W}(\mathrm{z})$ has converged, $\mathrm{E}(\mathrm{z})=0$. Equation

$$
\mathrm{W}(\mathrm{z})=-\mathrm{P}(\mathrm{z})
$$

Secondary -path effects: The error signal $\mathrm{e}(\mathrm{n})$ is measured at the error microphone downstream of the canceling speaker. Where the primary noise $d(n)$ is combined with the anti-noise $y(n)$ output from the adaptive filter. The anti-noise signal can be modified by the secondary-path function $\mathrm{H}(\mathrm{z})$ in the acoustic channel from $y(n)$ to $e(n)$, just as the primary noise is modified by the primary path $\mathrm{P}(\mathrm{z})$ from the noise source to the error sensor. Therefore, it is necessary to compensate for $\mathrm{H}(\mathrm{z})$. The z-transform of error signal $\mathrm{e}(\mathrm{n})$ is:

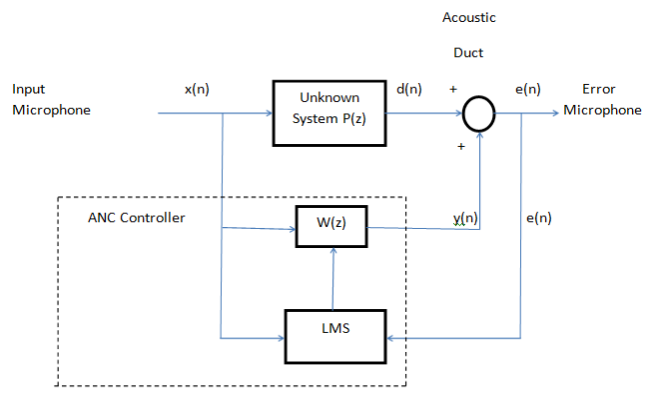

Fig.4.Block Diagram of ANC System modified to include $\mathrm{H}(\mathrm{Z})$ Assuming that $\mathrm{W}(\mathrm{z})$ has sufficient order, after the convergence of the adaptive filter, the residual error is zero (that is, $\mathrm{E}(\mathrm{z})=0$. This result requires $\mathrm{W}(\mathrm{z})$ to be:

$$
\mathrm{W}(\mathrm{Z})=-\frac{P(Z)}{H(Z)}
$$

However, it is impossible to invert the inherent delay caused by $\mathrm{H}(\mathrm{z})$ if the primary path $\mathrm{P}(\mathrm{z})$ does not contain a delay of at least equal length. This is the overall limiting causality constraint in broadband feed forward control systems. The FXLMS algorithm is illustrated in Fig.5, where the output $\mathrm{y}(\mathrm{n})$ is computed as:

$$
\mathrm{Y}(\mathrm{n})=\mathrm{w}^{T}(\mathrm{n}) \mathrm{x}(\mathrm{n})=\sum_{i=0}^{n-1} w_{i}(n) x(n-i)
$$

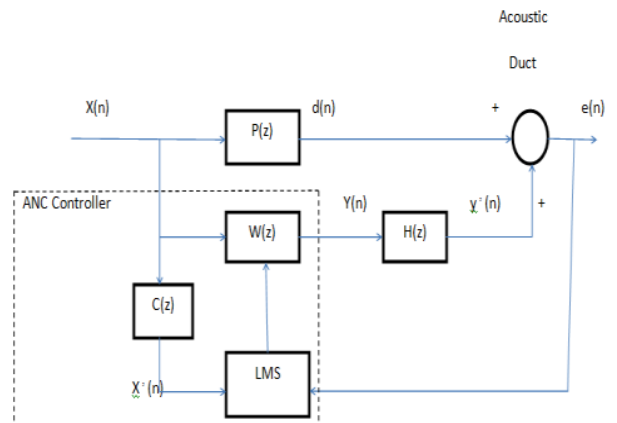

Fig.5. Block Diagram of the FXLMS Algorithm for ANC

The FXLMS algorithm can be expressed as:

$$
\mathrm{w}(\mathrm{n}+1)=\mathrm{w}(\mathrm{n})-\mu \mathrm{e}(\mathrm{n}) \mathrm{x}(\mathrm{n}) \mathrm{h}(\mathrm{n})
$$

Where $\mu$ is the step size of the algorithm that determines the stability and convergence of the algorithm and $h(n)$ is the impulse response of $\mathrm{H}(\mathrm{z})$. Therefore, the input vector $\mathrm{x}(\mathrm{n})$ is filtered by $\mathrm{H}(\mathrm{z})$ before updating the weight vector.

\section{MATLAB SIMULATIONS OF ADAPTIVE ALGORITHMS}

Each of the adaptive algorithms outlined in Section-III were implementing using MATLAB. Adaptive algorithms were implemented without window function and with different window functions. The fallowing simulation results shows the desired signal, adaptive output, estimation error signal and their actual filter \& estimated coefficients for adaptive algorithms i.e. LMS Algorithm, Normalized LMS Algorithm and Filtered-X LMS Algorithm with vocal input with total iterations 26432 and sampling frequency 16000, FIR filter with order of 26432, step size parameter $=0.008$. The Mean Square Error shows that as the algorithm progresses the average value of the cost function decreases, this corresponds to the adaptive filters impulse response converge ring to the actual impulse response. The success of the cancellation can be determined by calculating the ratio of the desired signal and error signal, attenuation and root mean square value. 

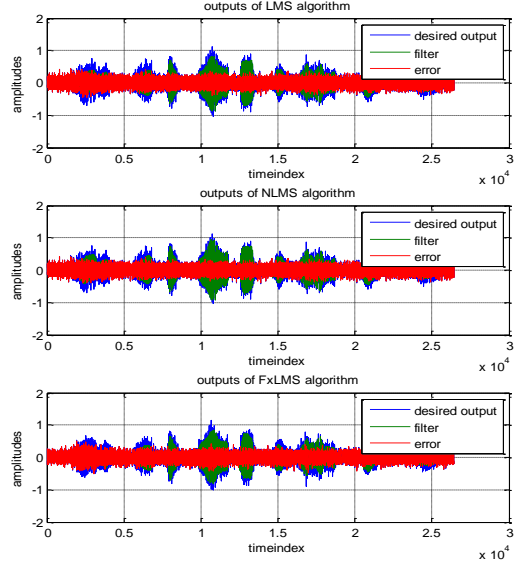
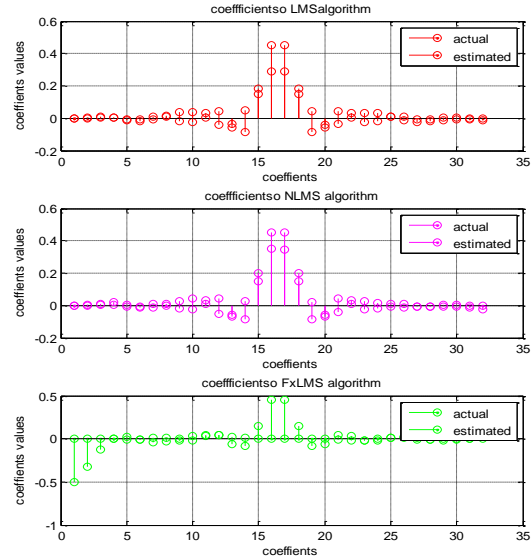

Fig.6. Desired signal, adaptive output, estimation error signal and filter coefficients for adaptive algorithms without window functions
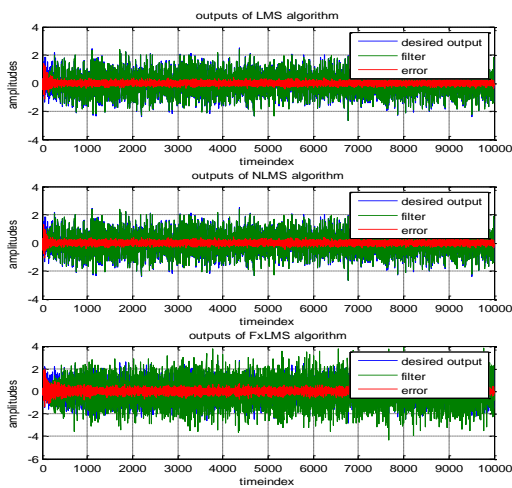
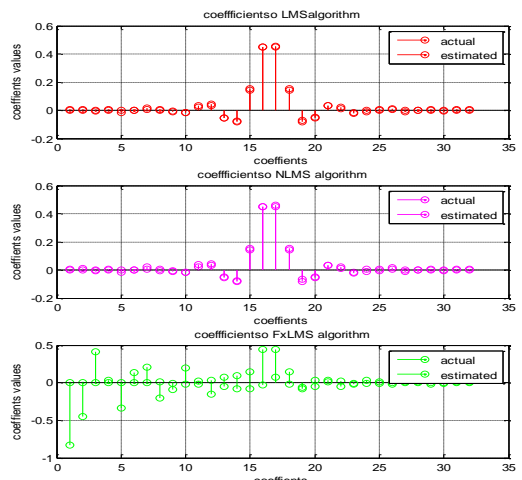

Fig.7. Desired signal, adaptive output, estimation error signal and filter coefficients for adaptive algorithms with Blackman window function
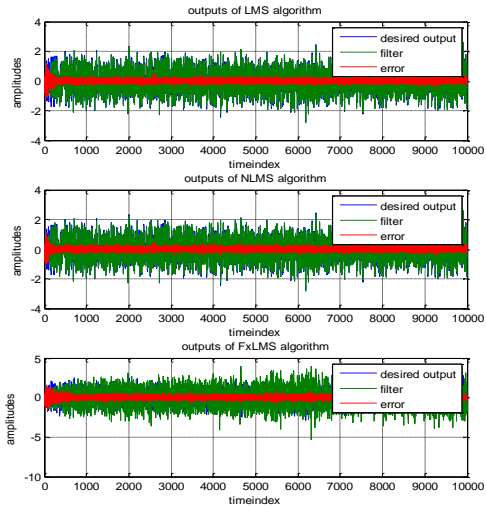
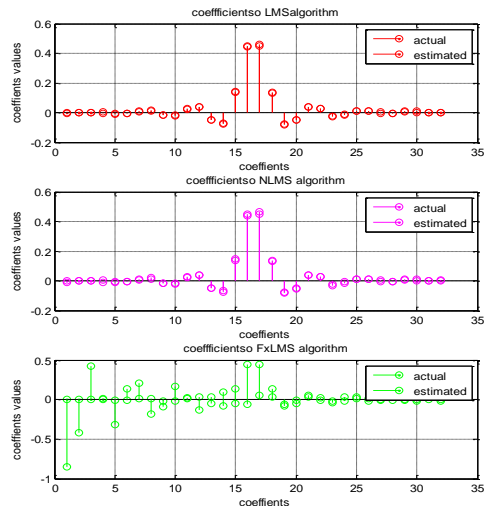

Fig.8. Desired signal, adaptive output, estimation error signal and filter coefficients for adaptive algorithms with Bartlett window function 

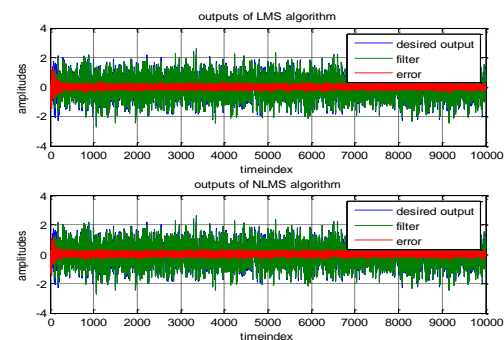

touputs of FxLMS algorith

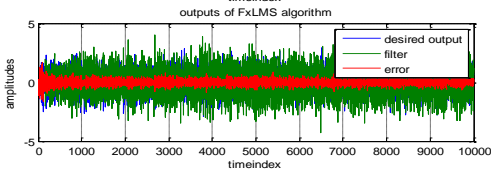

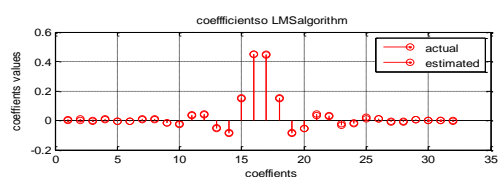
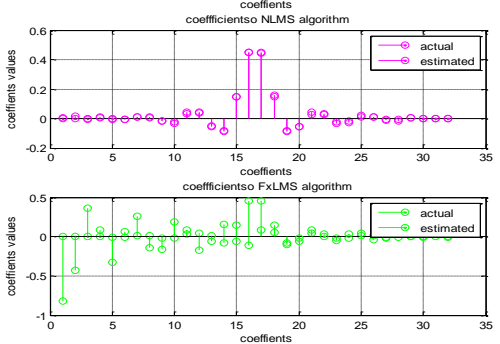

Fig.9. Desired signal, adaptive output, estimation error signal and filter coefficients for adaptive Algorithms with hamming window function

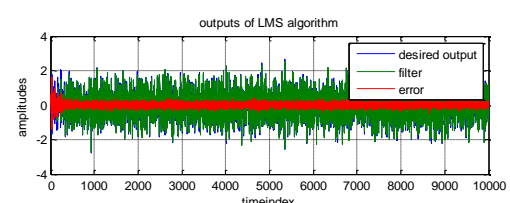

outputs of NLMS algorithm

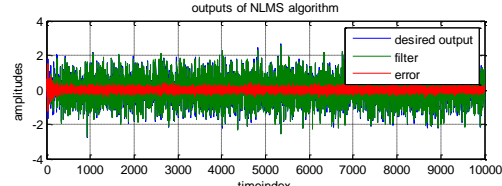
outputs of FxLMS algorithm

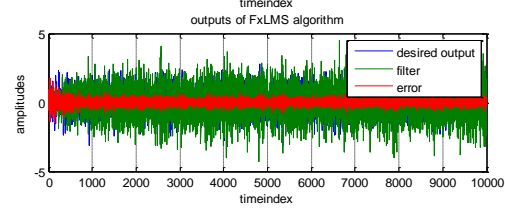

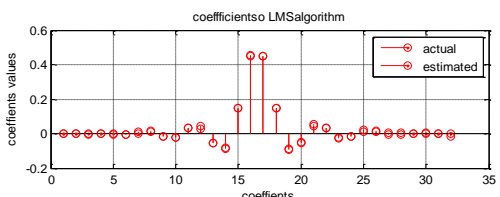
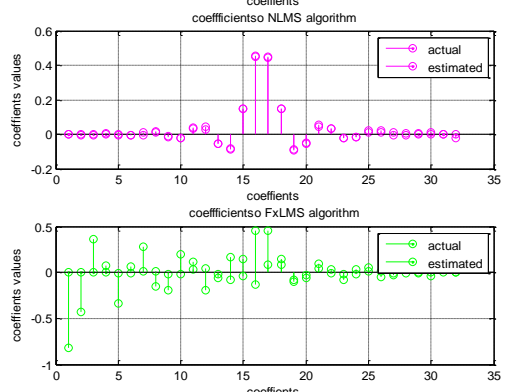

Fig.10. Desired signal, adaptive output, estimation error signal and filter coefficients for adaptive algorithms with Hanning window function

A summary of the performance of the adaptive filtering algorithms is expressed in Table is shown in below Fig.16.

\begin{tabular}{|c|c|c|c|c|c|c|c|c|c|}
\hline \multirow{2}{*}{ TYPE } & \multicolumn{3}{|c|}{ LMS } & \multicolumn{3}{c|}{ NLMS } & \multicolumn{3}{c|}{ FxLMS } \\
\cline { 2 - 10 } & SNR & DISTORTION & RMS & SNR & DISTORTION & RMS & SNR & DISTORTION & RMS \\
\hline $\begin{array}{c}\text { Without } \\
\text { window }\end{array}$ & 4.9769 & -19.5672 & 0.1051 & 5.1433 & -19.7335 & 0.1031 & 4.7728 & -19.3841 & 0.1073 \\
\hline $\begin{array}{c}\text { Blackman } \\
\text { window }\end{array}$ & 15.0943 & -18.3218 & 0.1213 & 15.1412 & -18.3687 & 0.1207 & 11.2977 & -13.6957 & 0.2066 \\
\hline $\begin{array}{c}\text { Bartlett } \\
\text { window }\end{array}$ & 15.0457 & -18.2743 & 0.1220 & 15.0930 & -18.3216 & 0.1213 & 10.9395 & -13.3559 & 0.2149 \\
\hline $\begin{array}{c}\text { Hamming } \\
\text { window }\end{array}$ & 15.0920 & -18.2078 & 0.1229 & 15.0918 & -18.2076 & 0.1229 & 10.4553 & -12.7829 & 0.2295 \\
\hline $\begin{array}{c}\text { Hanning } \\
\text { window }\end{array}$ & 14.9130 & -18.2109 & 0.1229 & 14.9793 & -18.2773 & 0.1219 & 10.4546 & -12.9383 & 0.22255 \\
\hline
\end{tabular}

\section{V.CONCLUSION}

Adaptive algorithms were implemented without window function and with different window functions. The fallowing simulation results shows the desired signal, adaptive output, estimation error signal and their actual filter \& estimated coefficients for adaptive algorithms i.e. LMS Algorithm, Normalized LMS Algorithm and Filtered-X LMS Algorithm with vocal input with total iterations 26432 and sampling frequency 16000, FIR filter 
with order of 26432, step size parameter $=0.008$. The Mean Square Error shows that as the algorithm progresses the average value of the cost function decreases. This corresponds to the adaptive filters impulse response converge ring to the actual impulse response. The success of the cancellation can be determined by calculating the ratio of the desired signal and error signal, attenuation and root mean square value. It can be seen that when considering the algorithms with window functions are performing better results than without window techniques. It is also noticed that the SNR, Distortion characteristics and attenuation values are better when we using Hanning window function. It is also noticed that FxLMS algorithm is suitable for real time active cancellation system.

\section{REFERENCES}

1. Nelson, P. A., and S. J. Elliott, Active Control of Sound, Academic Press, San Diego, CA, 1992.

2. Lueg, P., "Process of Silencing Sound Oscillations," U.S. Patent No. 2,043,416, June, 1936.

3. Burgess, J. C., "Active Adaptive Sound Control in a Duct: A Computer Simulation," J. Acoust. Soc. Am., Vol. 70, No. 3, Sept. 1981, pp. 715-726.

4. Widrow, B., and S. D. Stearns, Adaptive Signal Processing, PrenticeHall, Englewood Cliffs, NJ, 1985.

5. Kuo, S. M., and C. Chen, "Implementation of Adaptive Filters with the TMS320C25 or the TMS320C30," Digital Signal Processing Applications with the TMS320 Family, Volume 3, edited by P. Papamichalis, Prentice-Hall, Englewood Cliffs, NJ, 1990, pp. 191271.

6. Olson, H. F., and E. G. May, "Electronic Sound Absorber," J. Acoust. Soc. Am., Vol. 25, No. 6, Nov. 1953, pp. 1130-1136.

7. Olson, H. F., "Electronic Control of Noise, Vibration, and Reverberation," J. Acoust. Soc. Am., Vol. 28, No. 5, 1956. pp. 966972 .

8. Morgan, D. R., "A Hierarchy of Performance Analysis Techniques for Adaptive Active Control of Sound and Vibration," J. Acoust. Soc. Am., Vol. 89, No. 5, May, 1991, pp. 2362-2369.

9. Nishimura, M., "Some Problems of Active Noise Control for Practical Use," Proc. Int. Symp. Active Control of Sound and Vibration, Tokyo, 1991, pp. 157-164.

10. Roure, A., "Self-Adaptive Broadband Active Sound Control System," J. of Sound and Vibration, Vol. 101, No. 3, 1985, pp. 429-441. 